\title{
Strong color fields and heavy flavor production
}

\author{
I. Bautista and C. Pajares*
}

December 19, 2018

\begin{abstract}
The clustering of color sources provides a natural framework for soft partonic interactions producing strong color fields. We study the consequences of these color fields in the production of heavy flavor and the behavior of the nuclear modification factor.
\end{abstract}

\section{Introduction}

Heavy flavor production in heavy ions collisions is an ideal probe to study the early time dynamics of these nuclear collisions. Several theoretical studies predict [1] a substantial enhancement of open charm production associated to deconfined parton matter relative to the case of a purely hadronic scenario without quark-gluon plasma formation. Recent studies point out that the dynamics of heavy quarks is dominated by partonic interactions in a strongly coupled plasma modeled neither by hadronic interactions nor by color screening alone [2]. Therefore, these quarks are very

*IGFAE and Departamento de Física de Partículas, Univ. of Santiago de Compostela, 15706, Santiago de Compostela, Spain relevant in the study of the initial state of the collision. Owing to difficulties in reconstruct the $D$-mesons decay vertex, RHIC experiments have measured open charmed quarks indirectly, via the semileptonic decay to nonphotonic electrons or muons [3] [4]. In the standard picture charm quarks are produced by initial gluon fusion and their production rates are expected to be well described by perturbative quantum chromodynamics (pQCD) at fixed order plus next-to-leading logarithms (FONLL) [5]. The suppression of single, nonphotonic electrons or muons at RHIC is usually attributed to heavy-quark energy loss. As a charmed quark of energy $E$ cannot radiate gluons forming an angle below $\arcsin (m / E)$ (dead cone effect), it is expected that heavy quarks lose less energy than light quarks [6], but the suppression experimentally observed is similar. In fact, many calculations based on energy loss via hard scattering [7] or via multiple soft collisions [8] obtained less suppression than the experimental data when the beauty contribution is taken into account. Similar results are obtained in evaluations based on medium interactions or collisional dissociation [9]. However, it has 
been argued [10] [11] that under the assumption of an enhancement of the heavy-quark baryon-to-meson ratio, analogous to the case of the proton-to-pion and the $\Lambda$-to-kaon ratios measured in $\mathrm{Au}-\mathrm{Au}$ collisions at $\mathrm{RHIC}$, it is possible to achieve a larger suppression of the nuclear modification factor. This is possible, because the heavy-quark mesons have a larger branching ratio to decay inclusively into electrons as compared to heavyquark baryons and therefore, when the former are less copiously produced in a heavyion environment, the nuclear modified factor decreases, even in the absence of heavyquark energy loss. Indeed the single nonphotonic nuclear modified factor, $R_{A A}^{e}$ can be expressed as [12] $R_{A A}^{e}=R_{A A}^{D+\Lambda} F$ where $R_{A A}^{D+\Lambda}$ is the nuclear modified factor for $\mathrm{D}$ and $\Lambda_{c}$, i.e.

$$
R_{A A}^{D+\Lambda}=\frac{N_{A A}^{D}+N_{A A}^{\Lambda}}{N_{\text {coll }}\left(N_{p p}^{D}+N_{p p}^{\Lambda}\right)}
$$

with $N^{D}$ and $N^{\Lambda}$ the produced $D$ and $\Lambda$ in $A A$ or $p p$ collisions and the $N_{\text {coll }}$ is the number of collisions at a given centrality. The factor $F$ is given by the expression

$$
F=\frac{(1+a)(1+x C a)}{(1+C a)(1+x a)}
$$

where, $a$ and $C a$ are the charmed baryon-tomeson ratios in proton-proton and $A A$ collisions respectively. Therefore $C$ represents the enhancement factor for the ratio of charm baryons to mesons in $A A$ as compared to $p p$ collisions, $x$ is the ratio between the branching ratios for the inclusive decay of $\Lambda$ and $D$ into electrons:

$$
a=\left(\frac{\Lambda}{D}\right)_{p p}, C a=\left(\frac{\Lambda}{D}\right)_{A A}, x=\frac{B^{\Lambda \rightarrow e}}{B^{D \rightarrow e}}
$$

In [12] $x$ has been estimated to be 0.14. As long as $C$ is larger than 1 the factor $F$ becomes less than 1 and $R_{A A}^{e}<R_{A A}^{D+\Lambda}$. The main question to solve is whether the expected charmed baryon-to-meson expected enhancement is large enough to explain the difference with the experimental data.

In a high-energy heavy-ion collision, strong color fields are expected to be produced between the partons of the projectile and target [13 14 [15]. These color fields are similar to those that appear in the glasma [16] produced in the color glass condensate (CGC). In a string heavy-quark pairs are produced via the Schwinger mechanism with a rate $\Gamma_{Q \bar{Q}}=\exp \left[-\frac{\pi m_{Q}^{2}}{k}\right]$ where $k$, is the effective string tension, proportional to the strength of the field (for a single string $k_{1} \sim 1 \mathrm{GeV} / \mathrm{fm}$ ). Longitudinal string models predict for heavy flavor a very suppressed production rate, since

$$
\frac{\Gamma_{Q \bar{Q}}}{\Gamma_{q \bar{q}}}=\exp \left(\frac{-\pi}{k_{1}}\left(m_{Q}^{2}-m_{q}^{2}\right)\right) \ll 1
$$

for $Q=c$ and $q=u, d$. The color in these strings is confined to a small area in the transverse space, $\pi r_{0}^{2}$, with $r_{0} \simeq 0.25 \mathrm{fm}$. In a central heavy-ion collision many strings are formed between the partons of the projectile and target in a limited collision area, starting to overlap each other, forming clusters. The field strength of the cluster is proportional to the square root of the number of strings. So, for a cluster of nine strings, the string tension increases more than eight orders of magnitude becoming comparable to the initial FONLLpQCD. The effect of strong 
color fields for open charm has been investigated before 13 showing that a three- fold increase of the effective string tension results in a sizable enhancement of the total charm cross section and the nuclear modified factor shows a suppression at moderate $p_{T}$ consistent with the RHIC data.

In this paper, we study the effects of strong color fields in the framework of percolation of strings [17]. In this framework, a strong color field is obtained inside the clusters formed by the overlapping of individual strings. The clusters behave like individual strings with a higher string tension owing to the higher color field, and their energy momentum is the sum of the energy-momenta of individual strings. The color field of the string is stretched between a quark and an antiquark or between a diquark and a quark located at the extremes of the string. In the case of a cluster, instead of quarks or antiquarks we have complexes $Q$ and $\bar{Q}$ formed from the different quarks and their antiquarks or diquarks and quarks at the extremes of the individual strings [18] [19]. The clusters behave like strings with complexes $Q \bar{Q}$, located at the end, decaying into new pairs $Q \bar{Q}, \bar{Q} Q$, until they come to objects with mass comparable to hadron masses, which are identified with the known hadrons by combining the produced quarks or antiquarks with the appropriate statistical weights. In this way, the production of baryons and antibaryons is enhanced with the number of strings in the cluster. The cluster not only has a stronger color field than the individual string giving rise to a mass-enhancement effect but also enhances the production of baryons relative to mesons owing to the increasing probability of getting three quarks or three antiquarks from the complex $Q \bar{Q}[18]$. This second effect is similar to what happens in coalescence or recombination models [20] [21].

The percolation of strings incorporates to some extent the recombination of flavors in a dynamical way. Indeed a dynamical quark recombination model has shown a sizable suppression factor for the nonphotonic electrons nuclear modification factor [12].

We evaluate the nuclear modification factor for $D_{0}, \Lambda_{c}$ and $\mathrm{B}$ at RHIC energies, computing also the baryon-to-meson ratio in $A A$ and $p p$ collisions. We observe also in $p p$ a moderate enhancement of the ratio as a function of the transverse momentum which has consequences concerning the value of $F$ and therefore the rate of the nonphotonic electron suppression. The plan of the paper is as follows: In the next section, we introduce briefly the percolation of the strings, and then we present our results and conclusions.

\section{The string percolation model}

In the string percolation model [17] [22] [23] [24] 25], multiparticle production is described in terms of color strings stretched between the partons of the projectile and the target. With increasing energy and/or atomic number of the colliding particles, the number of strings $N_{s}$, grows and they start to overlap forming clusters, very much like disks in two-dimensional percolation theory. 
At a certain critical density, a macroscopical cluster appears, which marks the percolation phase transition. This density corresponds to the value $\eta_{c}=1.2-1.5$ (depending on the profile function of the colliding nuclei) where $\eta=N_{s} S_{1} / S_{A}$ and $S_{A}$ stands for the overlapping area of the colliding objects. A cluster of $n$ strings behaves like a single string with the energy momentum corresponding to the sum of individual ones and with a higher color field corresponding to the vectorial sum in color space of the color fields of the individual strings. In this way, the mean multiplicity $<\mu_{n}>$ and the mean transverse momentum squared $<p_{T n}^{2}>$ of the particles produced by a cluster are given by

$$
<\mu_{n}>=\sqrt{\frac{n S_{n}}{S_{1}}}<\mu_{1}>
$$

and $\left\langle p_{T n}^{2}>=\sqrt{\frac{n S_{1}}{S_{n}}}<p_{T 1}^{2}>\right.$, where $<\mu_{1}>$ and $\left\langle p_{T 1}\right\rangle$ are the corresponding quantities in a single string.

In the limit of high density of strings, equations (5) transforms into 24

$$
<\mu>=N_{s} F(\eta)<\mu_{1}>
$$

,$<p_{T}^{2}>=\frac{\left\langle p_{T 1}^{2}>\right.}{F(\eta)}$ with $F(\eta)=\sqrt{\frac{1-e^{-\eta}}{\eta}}$.

For a specific kind of particle $i$, we will use: $<\mu_{1}>_{i},<p_{T 1}^{2}>_{i},<\mu_{n}>_{i}$, and $<p_{T n}^{2}>_{i}$ for the corresponding quantities. To compute the multiplicities, we must know $N_{s}$ and $\mu_{1}$ (so for a fixed centrality, knowing $N_{s}$ we deduce the density $\eta$ ). Up to RHIC energies, in the central rapidity region $N_{s}$ is approximately twice the number of collisions, $N_{\text {coll }}$. However $N_{s}$ is larger than $2 N_{\text {coll }}$ at
RHIC and LHC energies, in the same way as in nucleon-nucleon collisions. According to color exchange models, such as dual parton model or the quark gluon string model [26] 27], the number of produced strings $N_{s}$ is larger than two, starting at the RHIC energies. Indeed, at high enough energy the strings are stretched not only between the diquarks(quarks) and quarks(diquarks) of the projectile and target respectively, but also between quarks(antiquarks) and antiquarks(quarks) of the sea. As the energy increases, more $q-\bar{q}$ or $\bar{q}-q$ are formed and $N_{s}$ becomes larger than two. For the same reason in $A A$ collisions, $N_{s}$ at high energy is larger than $2 N_{\text {coll }}$. In this work we take the values of $N_{s}$ from a Monte-Carlo based on the quark-gluon string model [28].

Concerning the transverse momentum distribution, one needs the distribution $g\left(x, p_{T}\right)$ for each cluster, and the mean square transverse momentum distribution of the clusters $W(x)$, where $x$ is the inverse of the mean of the squared transverse momentum of each cluster which is related to the cluster size by equation $(5)$. We take $g\left(x, p_{T}^{2}\right)=\exp \left(-p_{T}^{2} x\right)$ as it is used for fragmentation of the Lund string. For the weight function $W(x)$ we take the gamma distribution. The generalized gamma distributions are unique distributions stable under the cluster-size transformations 222] 29] 30]; for simplicity we choose gamma distribution simplicity 22 .

$$
W(x)=\frac{\gamma(\gamma x)^{k-1}}{\Gamma(k)} \exp (-k x)
$$

with

$$
\gamma=k /<x>
$$


and

$$
\frac{1}{k}=\frac{<x^{2}>-<x>^{2}}{<x>^{2}}
$$

The function $k$ measures the width of the distribution (7) and is the inverse of the normalized dispersion of the transverse momentum squared. The function $k$ depends on the density of strings $\eta$.

The transverse momentum distribution $f\left(p_{T}, y\right)$ of particle $i$ is

$$
f\left(p_{T}, y\right)=\frac{d N}{d p_{T}^{2} d y}=\int_{0}^{\infty} d x W(x) g\left(p_{T}, x\right)=
$$

$$
\frac{d N}{d y} \frac{k-1}{k} F(\eta) \frac{1}{\left(1+F(\eta) p_{T}^{2} / k<p_{T}^{2}>1 i\right)^{k}}
$$

The formula (10) is valid for all types of collisions, energies and also all kind of flavors. Later we will extend (10) for baryons. The function $k(\eta)$ was determined by comparing (10) to RHIC data. The function $k$ decreases with $\eta$ up to values $\eta \simeq 1$ (peripheral Au-Au collisions at RHIC energies) from there it increases slowly. This behavior was expected. In fact, at low density there is no overlapping of strings and there are isolated strings; therefore $k \rightarrow \infty$. When the density and therefore the numerator of Eq. (9) increases then $\mathrm{k}$ decreases. The minimum of $\mathrm{k}$ will be reached where the fluctuations in the cluster-size reach its maximum. Above this point, increasing $\eta$ these fluctuations decrease and $k$ increases. The agreement with data for $p_{T}$ up to $5 \mathrm{GeV} / \mathrm{c}$ is very good [22] 23$]$.

In percolation of strings the fragmentation of a cluster of many strings is via the Schwinger mechanism, producing successive pairs $Q \bar{Q}$, where $Q$ represents the complexes of quarks, diquarks and antiquarks at the extremes of the original string. It is clear that formula (10) only contains the effect of the stronger color field of the cluster, which enhances heavy particles production, irrespective of their being mesons or baryons, but it does not contain the breaking via flavor complexes $Q \bar{Q}$ and therefore cannot describe baryons correctly. In previous papers [18] [19] 28] Monte Carlo codes were presented where this mechanism was built up, but with the approximation of fusion of only two strings [17] [18] or using an effective color field [28]. To to keep a closed analytical formula, incorporating the antibaryon and baryon enhancement from the mechanism depicted here, we observe that this enhancement is similar to using the formula (10) with a larger density, or equivalently with a larger $N_{s}$. This means that for antibayons or baryons if we want to continue with formula (10) we must replace $\eta$ by $\bar{\eta}_{B}$,

$$
\bar{\eta}_{\bar{B}}=N_{s}^{\alpha} \eta
$$

and instead of the first equation (6) we must use

$$
\mu_{\bar{B}}=N_{s}^{1+\alpha} F\left(\eta_{B}\right) \mu_{1 \bar{B}}
$$

where the parameter $\alpha$ is obtained from a fit to the experimental dependence of the $p_{T^{-}}$ integrated $\bar{p}$ spectra with centrality [31]. The obtained value is $\alpha=0.09$. In same sense we can say that the antibaryons (baryons) probe a higher density than mesons for a fixed energy and type of collisions.

The equations (10), (11) and (12) allow us to compute the antibaryon (baryon) spectra. The equations (11) and (12) replace the recombination process described in this section 
and it should be considered as an approximation to keep the analytical formula (10). The formulae (10), (11) and (12) are valid for all kind of particles and not only for heavy flavor. We will show some results concerning light flavor.

\section{Results}

The equation (10) is limited to low and moderate $p_{T}$ not higher than $4-5 \mathrm{GeV} / \mathrm{c}$. In fact, we consider a gaussian $p_{T}$ distribution for the particles produced from the fragmentation of a string, without any power-like tail. This excludes the high- $p_{T}$ behavior, although our formula (10) allows for an interpolation from low to high $p_{T}$. By continuity, the high $p_{T}$ suppression observed at RHIC should give rise to a suppression at moderate $p_{T}$, say $4-5$ $\mathrm{GeV} / \mathrm{c}$, which is the limit where our equations apply.

To know the $p_{T}$ distributions given by formula (6) we need the values of $\left\langle p_{T}^{2}>_{1 D} \simeq<\right.$ $p_{T}>_{1 D}^{2}$ and $<p_{T}^{2}>_{1 \Lambda_{c}} \simeq<p_{T}>_{1 \Lambda_{c}}^{2}$, i. e. the mean $p_{T}$ of $D$ and $\Lambda_{c}$ particles produced from one string. We use $<p_{T}>_{1 D}=1.5$ $\mathrm{GeV} / \mathrm{c}$ and $<p_{T}^{2}>_{1 \Lambda_{c}}=1.9 \mathrm{GeV} / \mathrm{c}$. The difference between these two values is close to the difference between the masses of $D_{0}$ and $\Lambda_{c}$ and also agrees with the difference between the values commonly used of primordial transverse momentum of pions and protons, $<p_{T}>_{1 \pi}=0.2-0.3 \mathrm{GeV} / \mathrm{c},<$ $p_{T}>_{1 p}=0.6-0.7 \mathrm{GeV} / \mathrm{c}$. For $\mathrm{B}$ we use $<p_{T}>_{1 B}=4.25 \mathrm{GeV} / \mathrm{c}$.

In formula (10) the normalization is established by the values of $\frac{d N}{d y}$ at $p_{T}=0$,

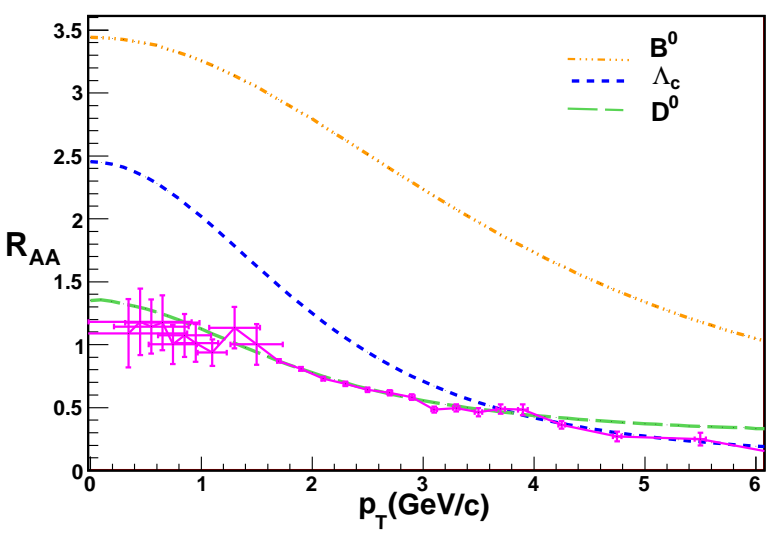

Figure 1: $R_{A A}$ for $\mathrm{Au}+\mathrm{Au}$ central collisions, bars is data taken from PHENIX [31].

which are computed using the formulae (6) for $\mathrm{D}$ and (9) for $\Lambda_{c}$. To do this, we use the values $\mu_{1 D}=\exp \left(-F(\eta) \frac{m_{D}^{2}}{\left\langle p_{T}>_{1 D}^{2}\right.}\right) \mu_{1 \pi}$ and $\mu_{1 \Lambda_{c}}=\exp \left(-F\left(\eta_{\Lambda_{c}}\right) m_{\Lambda_{c}}^{2} /<p_{T}>_{1 \Lambda_{c}}^{2}\right) \mu_{1 \pi}$, with $\mu_{1 \pi}=0.8$ [25]. We use these functions for $\mu_{1 D}$ and $\mu_{\Lambda_{c}}$ because for heavy particles, $m_{T}^{2}$ is very different from $p_{T}^{2}$. Concerning the function $k(\eta)$, we take the shape and values from the studies done in reference [25] for $A A$ collisions. In the case of $p p$ we take $k(\eta)=3.97$ at $\sqrt{s}=200 \mathrm{GeV}$ and $k(\eta)=4.07$ at $\sqrt{s}=5.5 \mathrm{TeV}$. We discuss later the sensibility of the obtained result for the ratio $\left(\Lambda_{c} / D^{0}\right)$ to different $\mathrm{k}$ values.

In fig. 1 we present our results for the nuclear modified factor $R_{A A}$ for $\mathrm{Au}-\mathrm{Au}$ collisions at RHIC for $D^{0}, \Lambda_{c}, B$ and $R_{A A}^{e}$ using formulae (1) as a function of $p_{T}^{2}$ compared with the experimental data on nonphotonic electrons.

The overall normalization is given by the 
value of $R_{A A}$ at $p_{T}^{2}=0$ which has to do with the factor $\exp \left(F\left(\eta_{p p}\right)-F\left(\eta_{A A}\right)\right) \frac{m_{D}^{2}}{\left\langle p_{T}^{2}>_{1 D}\right.}$. Since we know the number of strings produced in $p p$ and $A A$ collisions, we know $\eta_{p p}$, $\eta_{A A}, F\left(\eta_{A A}\right)$ and $F\left(\eta_{p p}\right)$ and the only free parameter is $<p_{T}^{2}>_{1 D}$. From the data we obtain $<p_{T}>_{1 D} \sim 1.5 \mathrm{GeV} / \mathrm{c}$. The experimental errors allow us a $15 \%$ freedom in the value of $<p_{T}>_{1 D}$, however a higher value than $1.5 \mathrm{GeV} / \mathrm{c}$ would not be realistic an a lower value will give rise to a higher normalization and therefore $R_{A A}$ for $p_{T}>4 \mathrm{GeV} / \mathrm{c}$ will exceed the experimental data even more than with the used value. The value of $R_{A A}$ for $D^{0}$ at low $p_{T}$ agrees with the results in [14] 32, for $p_{T}>4 \mathrm{GeV} / \mathrm{c}$ we obtain an $R_{A A}$ larger than the non-photonic leptonic data. In fig. 2, we present our results on $R_{A A}$ at $\sqrt{s}=5.5 \mathrm{TeV}$ for a $D^{0}, \Lambda_{c}$ and $\mathrm{B}$. We see that, as expected, as energy increases the low $p_{T} R_{A A}$ increases, although the suppression at intermediate $p_{T}$ is similar.

In fig 3 , we present the ratio $\Lambda_{c} / D^{0}$ for $\mathrm{Au}$ $\mathrm{Au}$ at $\sqrt{s}=200 \mathrm{GeV}$. We observe that the ratio increases up to a maximum of 1.45 around $p_{T} \sim 4-5 \mathrm{GeV} / \mathrm{c}$. A very similar enhancement has been obtained in the dynamical recombination model [12].

For comparison we include also our results for $\bar{p} / \pi$ at central $\mathrm{Au}-\mathrm{Au}$ collisions together with experimental data [33. In fig. 4 we show the ratio $\Lambda_{c} / D^{0}$ for $p p$ at $\sqrt{s}=200$ $\mathrm{GeV}$. We observe a very smooth enhancement. In fig 5 and fig. 6 we show our results for the ratio $\Lambda_{c} / D^{0}$ at $\sqrt{s}=5.5 \mathrm{TeV}$ for $\mathrm{Pb}-\mathrm{Pb}$ collisions and $p p$ collisions respectively. We observe in both of them larger

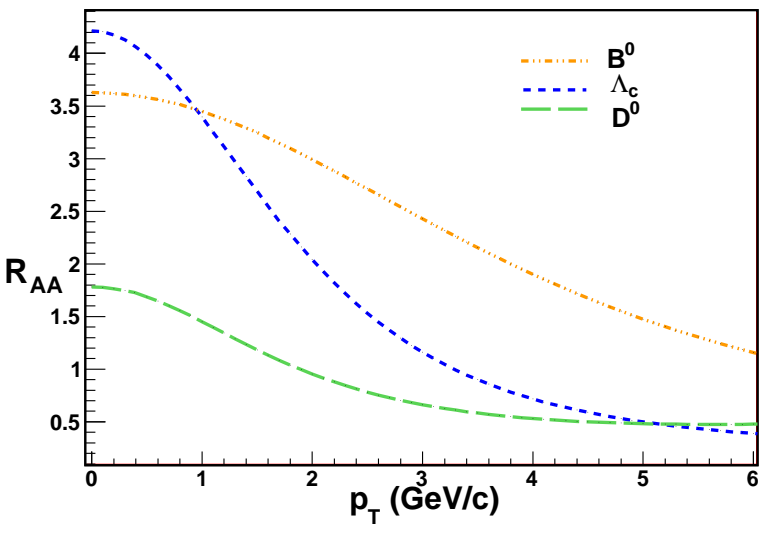

Figure 2: $R_{A A}$ for $\mathrm{Pb}+\mathrm{Pb}$ central collisions at $\sqrt{s}=5.5 \mathrm{TeV}$.

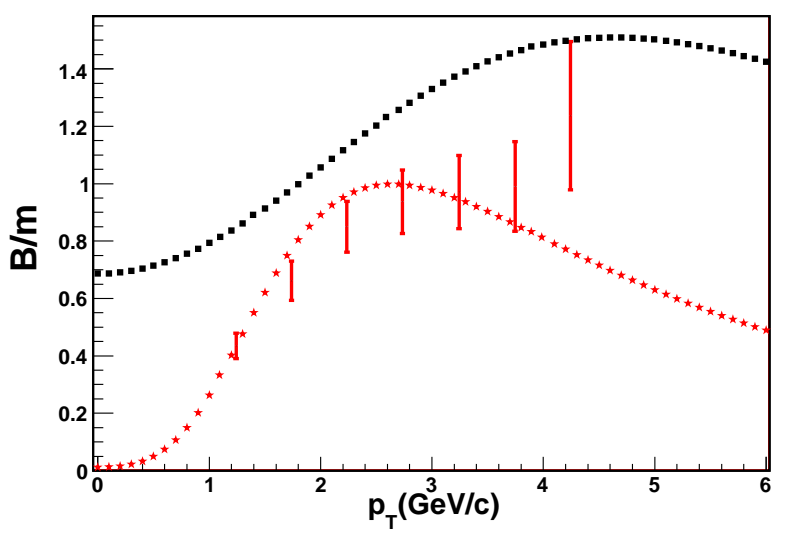

Figure 3: Squares are used for ratio $\Lambda_{c} / D^{0}$, starts are used for $\bar{p} / \pi$, and errorbars are used for data from PHENIX for Au-Au central collisions at $\sqrt{s}=200(\mathrm{GeV})$. 


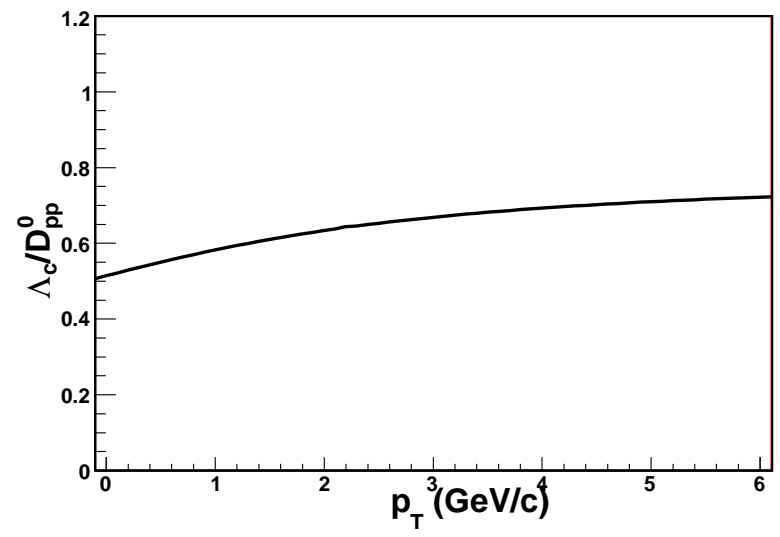

Figure 4: Ratio $\Lambda_{c} / D^{0}$ for $\mathrm{p}+\mathrm{p}$ collisions at $\sqrt{s}=200 \mathrm{GeV}$.

enhancement than at RHIC energies, particularly in the nuclear case. In fig. 7 we plot the factor $\mathrm{F}$ at $\sqrt{s}=200 \mathrm{GeV}$ (blue) and at $\sqrt{s}=5.5 \mathrm{TeV}$ (red line). We observe that at RHIC energies the factor $F$ is only slightly below one, and for $p_{T} \simeq 4-5 \mathrm{GeV} / \mathrm{c}$ it is clearly over 0.5 , which means that the $\Lambda_{c} / D^{0}$ enhancement in $A A$ is not able to explain all the difference between experimentally observed suppression of $R_{A A}$ for non-photonic electrons and the pQCD expectations. We have studied the effects due to the uncertainties in the $k$ values for $p p$ at this energy. For reasonable alternative $k$ values the enhancement of $\Lambda_{c} / D_{0}$ in pp with $p_{T}$ is larger giving rise to lower $\mathrm{C}$ factor in equation (3) and therefore the factor $\mathrm{F}$ is near to one, consistent with our main conclusion, namely that the $\Lambda_{c} / D^{0}$ enhancement is not able to explain all the difference between the experimentally observed values and the perturbative expectations.

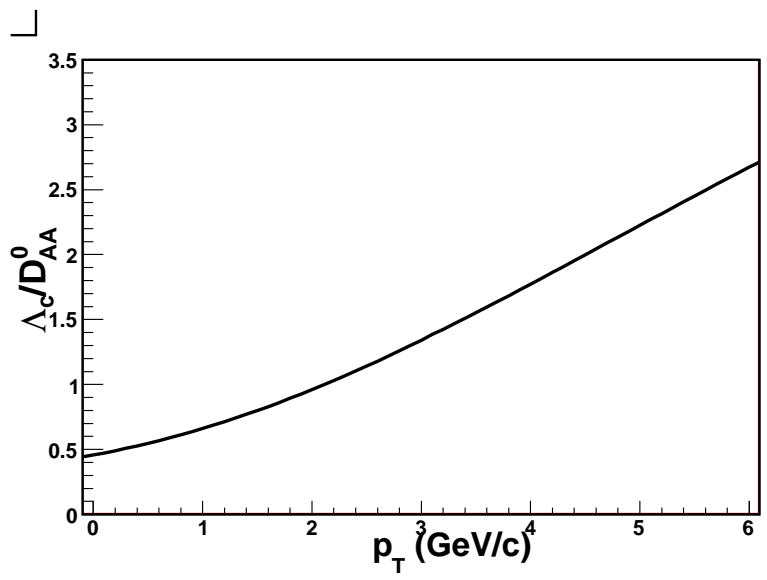

Figure 5: Ratio $\Lambda_{c} / D^{0}$ for $\mathrm{Pb}-\mathrm{Pb}$ central collisions at $\sqrt{s}=5.5 \mathrm{TeV}$.

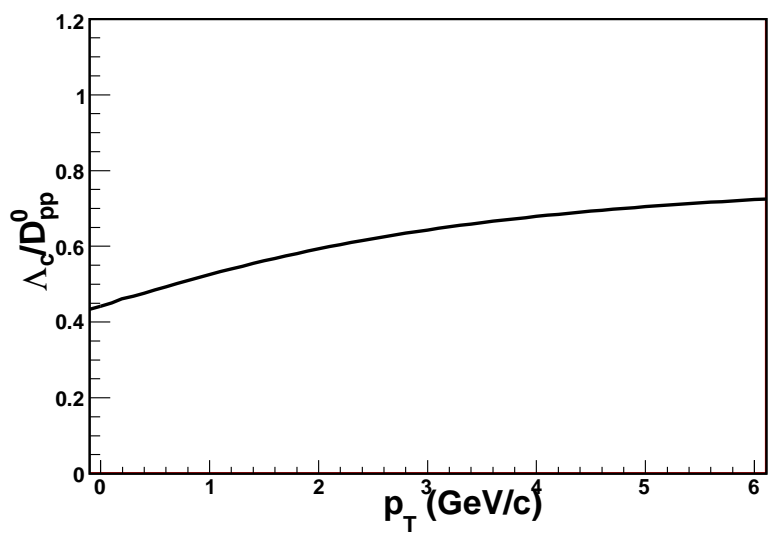

Figure 6: $R_{A A} \Lambda_{c} / D^{0}$ for $\mathrm{p}+\mathrm{p}$ collisions at $\sqrt{s}=5.5 \mathrm{TeV}$. 


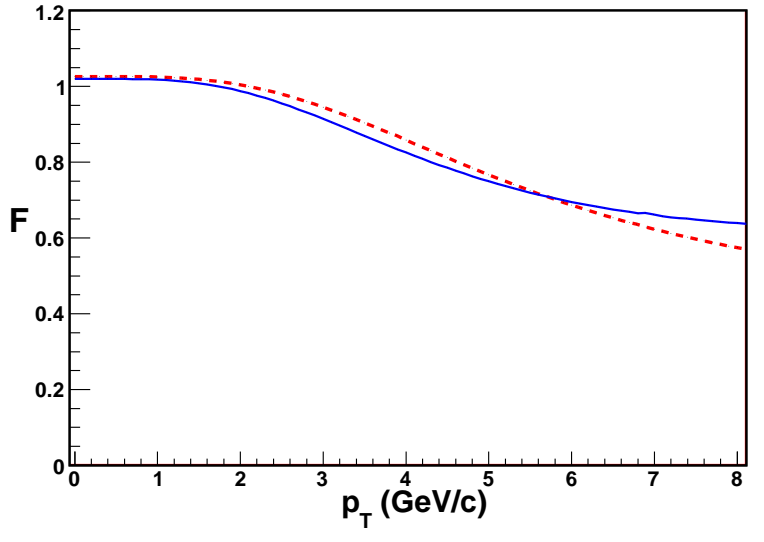

Figure 7: Factor $\mathrm{F}$ for central collisions at RHIC in full line and LHC energies in dashed lines.

\section{Conclusion}

The overlapping of the strings formed in the collision of heavy nuclei particles produces strong color fields which give rise to an enhancement of heavy flavor. We have computed the nuclear modification factor of $D^{0}$, $\Lambda_{c}$ and $B^{0}$ at RHIC and LHC energies for $A A$ collisions. Referring to $D^{0}$, we obtain a good agreement at low $p_{T}$ with the experimental data for the nuclear modification factor of non-photonic electrons. For $p_{T}$ values between 2 and $6 \mathrm{GeV} / \mathrm{c}$ our results obtained are over the experimental data as pQCD [18].

The ratio $\Lambda_{c} / D^{0}$ as a function of $p_{T}$ for $\mathrm{Au}$ $\mathrm{Au}$ collisions at $\sqrt{s}=200 \mathrm{GeV}$ is enhanced showing a maximum around $5 \mathrm{GeV} / \mathrm{c}$. Such as enhancement is much larger at LHC energies. However, the enhancement $\Lambda_{c} / D^{0}$ can explain only half of factor 2 difference between the experimental data and the pQCD expectations at RHIC energies.

In $\mathrm{p}$-p collisions the ratio $\Lambda_{c} / D^{0}$ also rises as a function of $p_{T}$ but very smoothly at RHIC energies. At LHC this increase is a factor of 2 between $p_{T}=0$ and $p_{T}=6 \mathrm{GeV} / \mathrm{c}$. The enhancements of $\Lambda_{c} / D^{0}$ in $\mathrm{AA}$ and $\mathrm{pp}$ collisions are larger at LHC than at RHIC as it was expected due to the stronger color fields produced.

\section{Acknowledgments}

We thank N. Armesto and A. Ayala and M. A. Braun for discussions. This research was supported by MICINN of Spain, under Grant FPA2008-01177 and by Xunta de Galicia.

\section{References}

[1] B. Muller, and X. N. Wang, Phys. Rev. Lett. 68, 2437 (1992); E. V. Shuryak, Phys. Rev. Lett. 68, 3270 (1992).

[2] O. Linnyk, E. L. Bratkovskaya and W. Cassing, Int. J. Mod. Phys. E 17, 1367 (2008).

[3] J. Adams et al. [STAR Collaboration], Phys. Rev. Lett. 94, 062301 (2005); B. I. Abelev et al. [STAR Collaboration], Phys. Rev. Lett. 98, 192301 (2007).

[4] S. S. Adler et al. [PHENIX Collaboration], Phys. Rev. Lett. 94, 082301 (2005); A. Adare et al. [PHENIX Collaboration], Phys. Rev. Lett. 98, 172301 (2007). 
[5] M. Cacciari, M. Greco and P. Nason, JHEP 9805, 007 (1998).

[6] Y. L. Dokshitzer and D. E. Kharzeev, Phys. Lett. B 519, 199 (2001).

[7] M. Djordjevic, M. Gyulassy, R. Vogt and S. Wicks, Phys. Lett. B 632, 81 (2006).

[8] N. Armesto, M. Cacciari, A. Dainese, C. A. Salgado and U. A. Wiedemann, Phys. Lett. B 637, 362 (2006).

[9] A. Adil and I. Vitev, Phys. Lett. B 649, 139 (2007).

[10] G. Martinez-Garcia, S. Gadrat and P. Crochet, Phys. Lett. B 663, 55 (2008); G. Martinez-Garcia, S. Gadrat and P. Crochet, J. Phys. G 35, 044023 (2008).

[11] D. C. Zhou, Z. Conesa del Valle, A. Dainese, H. T. Ding and G. Martinez Garcia, J. Phys. G 36, 064055 (2009).

[12] A. Ayala, J. Magnin, L. M. Montano, G. ToledoSanchez, Phys. Rev. C 80, 064905 (2009).

[13] V. ToporPop, M. Gyulassy, J. Barrette, C. Gale, S. Jeon and R. Bellwied, Phys. Rev. C 75, 014904 (2007).

[14] V. T. Pop, J. Barrette and M. Gyulassy, Phys. Rev. Lett. 102, 232302 (2009).

[15] N. Armesto, M. A. Braun, E. G. Ferreiro and C. Pajares, Phys. Lett. B 344, 301 (1995); J. Dias de Deus, E. G. Ferreiro, C. Pajares and R. Ugoccioni, Phys. Lett. B 581, 156 (2004).
[16] D. Kharzeev and K. Tuchin, Nucl. Phys. A 735, 248 (2004); L. McLerran, J. Phys. G 35, 104001 (2008).

[17] N. Armesto, M. A. Braun, E. G. Ferreiro and C. Pajares, Phys. Rev. Lett. 77, 3736 (1996)

[18] N. S. Amelin, M. A. Braun and C. Pajares, Z. Phys. C 63, 507 (1994). N. S. Amelin, M. A. Braun and C. Pajares, Phys. Lett. B 306, 312 (1993).

[19] N. S. Amelin, H. Stocker, W. Greiner, N. Armesto, M. A. Braun and C. Pajares, Phys. Rev. C 52, 362 (1995).

[20] R. C. Hwa and C. B. Yang, Phys. Rev. C 65, 034905 (2002) [Erratum-ibid. C 67, 059902 (2003)].

[21] R. J. Fries, B. Muller, C. Nonaka and S. A. Bass, Phys. Rev. Lett. 90, 202303 (2003).

[22] J. Dias de Deus, E. G. Ferreiro, C. Pajares and R. Ugoccioni, Eur. Phys. J. C 40, 229 (2005).

[23] C. Pajares, Eur. Phys. J. C 43, 9 (2005).

[24] M. A. Braun and C. Pajares, Phys. Rev. Lett. 85, 4864 (2000); M. A. Braun and C. Pajares, Eur. Phys. J. C 16, 349 (2000)

[25] L. Cunqueiro, J. Dias de Deus, E. G. Ferreiro and C. Pajares, Eur. Phys. J. C 53, 585 (2008) 
[26] A. Capella, U. Sukhatme, C. I. Tan and J. Tran Thanh Van, Phys. Lett. B 81 (1979) 68. A. Capella, U. Sukhatme, C. I. Tan and J. Tran Thanh Van, Phys. Rept. 236 (1994) 225. A. Capella, C. Pajares and A. V. Ramallo, Nucl. Phys. B 241 (1984) 75.

[27] A. B. Kaidalov and K. A. TerMartirosian, Phys. Lett. B 117, 247 (1982).

[28] N. S. Amelin, N. Armesto, C. Pajares and D. Sousa, Eur. Phys. J. C 22, 149 (2001); N. Armesto, C. Pajares and D. Sousa, Phys. Lett. B 527, 92 (2002).

[29] G. Jona-Lasinio, Nuovo Cim. B 26 (1975) 99.

[30] J. Dias de Deus, C. Pajares and C. A. Salgado, Phys. Lett. B 407, 335 (1997) A. Capella, J. Tran Thanh Van, R. Blankenbecler, C. Pajares and A. V. Ramallo, Phys. Lett. B 107, 106 (1981)

[31] PHENIX Collaboration, Phys. Rev. Lett. 88, 192303 (2002).

[32] M. G. Ryskin, A. G. Shuvaev and Yu. M. Shabelski, Phys. Atom. Nucl. 64, 120 (2001); [Yad. Fiz. 64, 123 (2001)]; C. Merino, C. Pajares, M. M. Ryzhinskiy, Yu. M. Shabelski, A. G. Shuvaev, arXiv:0910.2364 [hep-ph].

[33] S. S. Adler et al. [PHENIX Collaboration], Phys. Rev. C 69 (2004) 034909 